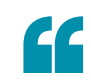

\section{tumours in} $\beta A d R^{\mathrm{KO}}$ mice stopped growing after

18 days and had reduced tumour vessel density

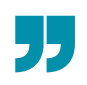

TUMOUR ANGIOGENESIS

\title{
Controlling nerves
}

Sympathetic nerves associate with tumours and promote tumorigenesis; however, the mechanisms behind this are poorly understood. New research published in Science shows that adrenergic innervation controls the angiogenic switch in prostate cancer by regulating vascular metabolism.

The researchers implanted prostate cancer xenografts orthotopically into mice lacking $\beta$-adrenergic receptors $A d r b 2$ and $A d r b 3\left(\beta \mathrm{AdR}^{\mathrm{KO}}\right)$. Whereas tumours in control mice $\left(\beta \mathrm{AdR}^{\mathrm{WT}}\right)$ grew exponentially, tumours in $\beta \mathrm{AdR}^{\mathrm{KO}}$ mice stopped growing after 18 days and had reduced tumour vessel density. $\beta \mathrm{AdR}^{\mathrm{WT}}$ mice treated with the neurotoxin 6-hydroxydopamine had reduced ability to recruit vasculature to an orthotopically transplanted collagen type I matrix that resembles the extracellular matrix in prostate cancer, suggesting that $\beta$-adrenergic signalling could regulate angiogenesis in prostate cancer.

To corroborate this finding, the researchers analysed tumours in the Hi-MYC spontaneous autochthonous prostate cancer mouse model. Upon overexpression of human $M Y C$, this model recapitulates the progression of prostate cancer from the low-grade pre-neoplastic stage to the high-grade malignant stage and, subsequently, invasive cancer. In high-grade tumours, they found increased nerve and vessel densities and higher levels of noradrenaline compared with lowgrade tumours. Deletion of Adrb2 in endothelial cells (ECs) of Hi-MYC mice (MYC;Adrb2 $\left.2^{\mathrm{ECKO}}\right)$, but not its deletion in other stromal cells, inhibited the progression from low-grade to high-grade tumours.

Functionally, ECs isolated from prostates of MYC;Adrb2 $2^{\mathrm{ECKO}}$ mice showed increased expression of mitochondrial metabolism genes compared with control ECs isolated from $M Y C ; A d r b 2^{\mathrm{WT}}$ mice, including cytochrome $c$ oxidase assembly factor 6 (Coa6), which is required for the assembly of the terminal enzyme of the electron transport chain. In addition, these ADRB2-deficient ECs had features consistent with increased oxidative phosphorylation activity, including a high mitochondrial membrane potential and high glucose uptake.

The researchers continued their functional analysis by using primary mouse prostate ECs that are capable of long-term survival through expression of the adenoviral E4ORF1 gene (E4ORF1+ ECs) and by using lentiviral transduction of short hairpin RNA (shRNA) that targets Adrb2. ADRB2-depleted E4ORF1 ${ }^{+}$ ECs grown in noradrenaline (NA)containing media were less able to migrate than, and had a similar oxidative phosphorylation profile to, ECs from $M Y C ; A d r b 2^{\mathrm{ECKO}}$ mice. Stable-isotope tracing analysis of $\left[{ }^{13} \mathrm{C}\right]$ glucose revealed an increased contribution of glucose to the tricarboxylic acid (TCA) cycle in ADRB2-depleted $E 4 O R F 1^{+}$ECs,

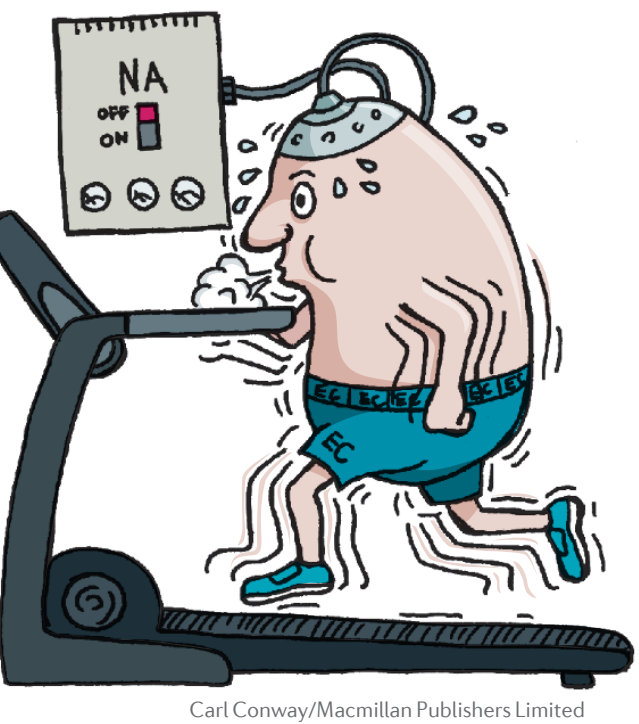

which was accompanied by increased oxygen consumption, production of mitochondrial ATP and expression of Coa6. When overexpressing Coa6 in $\mathrm{E} \mathrm{ORF1} 1^{+} \mathrm{ECs}$, oxidative phosphorylation was upregulated and the ability of these ECs to form vessels in co-transplanted prostate cancer xenografts was reduced. As the protein encoded by Coa 6 cooperates with cytochrome $c$ oxidase assembly protein 10 (encoded by Cox10), the researchers deleted Cox10 specifically in ECs of MYC;Adrb2 $2^{\mathrm{ECKO}}$ mice, which prevented the oxidative switch in ECs, rescued angiogenesis in prostate tumours and enabled cancer progression.

Overall, this study shows that angiogenesis in prostate cancer is promoted by adrenergic innervation, and this control is mediated by suppressing oxidative metabolism in ECs.

Ulrike Harjes

ORIGINAL ARTICLE Zahalka, A. H. et al. Adrenergic nerves activate an angio-metabolic switch in prostate cancer. Science 358, 321-326 (2017)

FURTHER READING Cole, S. W. et al.

Sympathetic nervous system regulation of the tumour microenvironment. Nat. Rev. Cancer 15 , 563-572 (2015) 